\title{
Aspectos
}

\section{Básicos de los}

Materiales

Usados en

Ingeniería

Jorge Alexander Aponte Rodríguez

Hernando A. Villazón Amarís*

\section{RESUMEN}

E l estudio de los materiales ha sido objeto De gran importancia en el desarrollo tecnológico a nivel mundial, pues gracias a éste la humanidad ha logrado fabricar armas para defenderse de las otras especies y herramientas para las labores agrícolas que nos dieron la posibilidad de tener civilizaciones sedentarias. Los materiales juegan un papel fundamental en el avance de la tecnología, y mediante el estudio y desarrollo de éstos, se han podido lograr mejores niveles de vida, fabricando automóviles, barcos, aviones, computadores y ahora ese fascinante y complejo mundo conectado por los modernos medios de comunicación.

Este artículo recopila algunos conceptos fundamentales acerca de los materiales para ingeniería, destacando la importancia y aplicaciones de estos en el mundo de hoy.

- Docentes del Departamento de Ingeniería Mecatrónica de la Universidad Militar "Nueva Granada". 


\section{TIPOS DE MATERIALES}

En ingeniería se habla convencionalmente de cinco tipos de materiales, estos son:

1. Metales y sus aleaciones: hasta hace relativamente poco tiempo (mediados del siglo $\mathrm{X})$, fueron los materiales de uso masivo para proyectos de ingeniería; y aunque hoy vienen siendo desplazados de muchos campos de aplicación por otros materiales, continuarán siendo una alternativa muy importante en la selección de materiales.

Algunos de los metales de uso corriente son: el hierro ( $\mathrm{Fe}$ ) el cual al igual que la mayoría de los metales no se utiliza solo, sino combinado con otro elemento denominado aleante y que para el caso del hierro es el Carbono, formando el compuesto (aleación) conocido como acero; el Oro $(\mathrm{Au})$ y la Plata $(\mathrm{Ag})$, así como el Cromo, el Níquel, el Vanadio y el Titanio, son apenas algunos ejemplos.

2. Cerámicos: son compuestos químicos con contenidos de elementos metálicos ( $\mathrm{Al}, \mathrm{Fe}$, $\mathrm{Zn}$, etc) y no metálicos ( $\mathrm{Si}, \mathrm{O}, \mathrm{N}, \mathrm{C})$. Algunos de los usualmente conocidos son: ladrillo, vidrio, porcelana, alúmina.

3. Polímeros: son esencialmente moléculas orgánicas $(\mathrm{C}$ e $\mathrm{H})$, organizadas en cadenas gigantes con elevados pesos moleculares. Algunos de los más utilizados son el polietileno (bolsas de la basura), cloruro de polivinilo P.V.C. (fabricación de tuberías), poliéster (empleado en algunas telas sintéticas), y el polietilenterftalato PET (como recipiente de gaseosas y bebidas).

4. Semiconductores: Son materiales que se comportan como aislantes eléctricos, pero que al cambiarles algunas condiciones como son la temperatura, presión, luz, o alguna otra condición física, cambian su comportamiento de aislantes a conductores eléctricos, lo que hace que encuentren su mayor campo de aplicación en la fabricación de componentes electrónicos tales como tarjetas y microchips, entre otros.

Presentan características muy precisas en cuanto a la conductividad eléctrica, que los hace indispensables en el desarrollo de muchos componentes de tipo electrónico. El Silicio, Germanio y Arseniuro de Galio GaAs son algunos ejemplos.

5. Materiales compuestos: Son fabricados mediante la combinación (no unión, ni mezcla) de dos o más materiales, buscando obtener propiedades que éstos no poseen de manera individual. El concreto, la fibra de vidrio, son materiales con las características mencionadas.

\section{PROPIEDADES DE LOS MATERIALES}

Pueden ser categorizadas en dos grupos.

1. Propiedades mecánicas: describen la forma como un material se comporta cuando está siendo sometido a la acción de cargas (fuerzas) o esfuerzos (fuerza por unidad de área). Las más importantes son:

1.1 Resistencia a la tracción: para determinarla se toma una probeta y se somete a la aplicación de carga como se muestra en la figura 1. Los resultados de ésta se grafican en una curva Esfuerzo vs Deformación (figura 2) y de esta manera se determinan algunas características particulares del material como son el esfuerzo de fluencia (esfuerzo al cual el material cambia su comportamiento de elástico a plástico), el esfuerzo máximo que puede soportar el material y el esfuerzo de rotura.

1.2 Rigidez: con base en la curva esfuerzo deformación se puede hallar una relación 


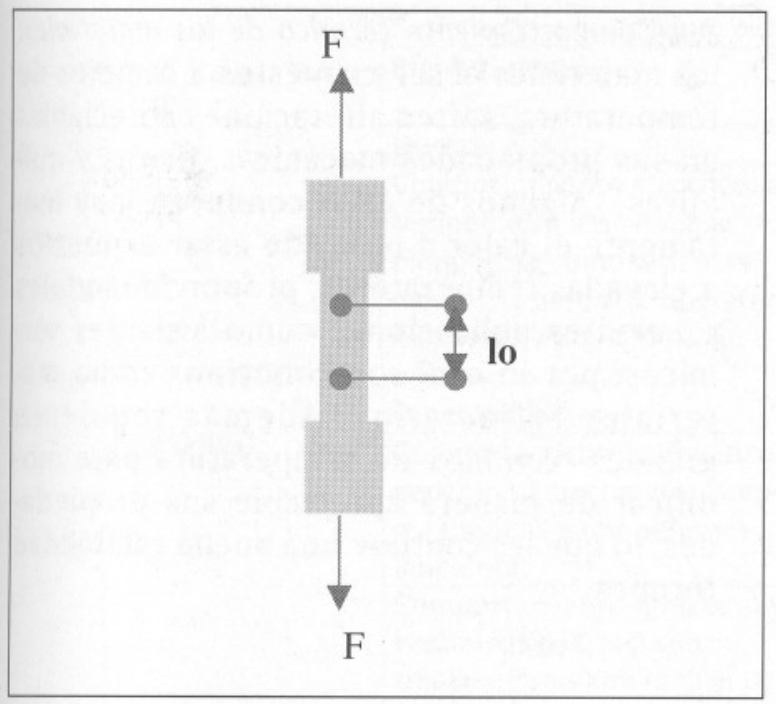

Figura 1. Probeta sometida a tracción

entre estas variables, denominada Módulo de Young; a medida que ésta se hace más grande se considera que el material es más rígido, ya que se necesitan esfuerzos muy grandes para lograr pequeñas deformaciones.

1.3 Ductilidad: tiene que ver con la capacidad que tiene un material para deformarse antes de romperse. Algunos materiales no admiten prácticamente ningún tipo de deformación antes de la ruptura, a estos se denominan materiales frágiles, mientras que aquellos que experimentan grandes deformaciones antes de la ruptura se denominan materiales dúctiles.

1.4 Dureza: Es la resistencia que opone un material a ser penetrado o rayado.

1.5 Tenacidad: Indica la capacidad que presenta el material a absorber energías de impacto (resistencia al impacto), entendiendo impacto como un golpe repentino que se proporciona al material. Los materiales dúctiles son de muy buena tenacidad, mientras que los rígidos (o frágiles) presentan baja resistencia al impacto.

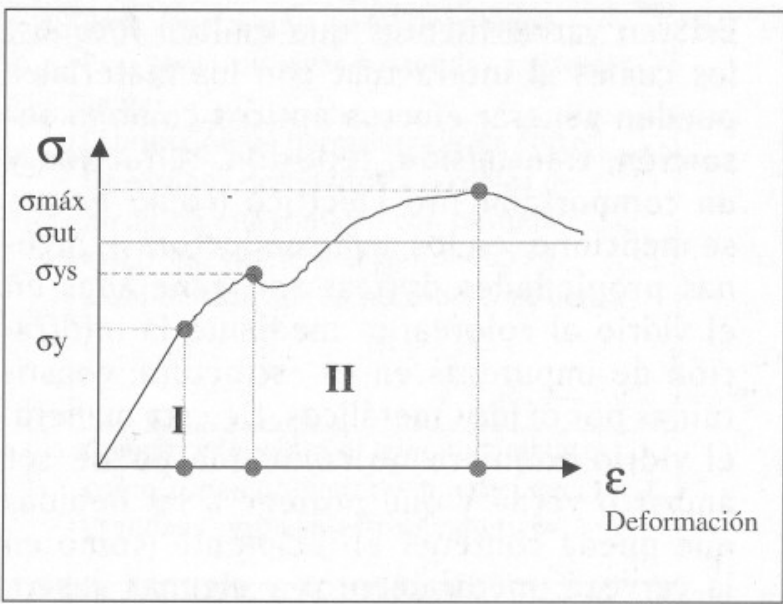

Figura 2. Gráfica del ensayo de resistencia a la tracción.

Donde: $\boldsymbol{F}=$ esfuerzo, $\boldsymbol{g}=$ deformación unitaria., Fmáx =esfuerzo máximo., Fut =esfuerzo último., Fys=esfuerzo de fluencia., I=zona de comportamiento elástico, $I I=$ zona de comportamiento plástico.

\section{Propiedades físicas:}

2.1 Comportamiento eléctrico de los materiales: algunos materiales permiten el paso de corrientes eléctricas a través de ellos, y por ésto son denominados conductores eléctricos; otros materiales no presentan esta característica y por eso son llamados aislantes eléctricos; existen otros materiales que bajo algunas condiciones especiales (temperatura, presión, luz, etc.) se pueden comportar como aislantes o conductores eléctricos y son conocidos como semiconductores.

2.2 Comportamiento magnético de los materiales: la mayoría de los materiales están expuestos a la interacción con campos magnéticos, y algunos pueden llegar a convertirse en materiales magnéticos o interferir en el comportamiento de éstos.

2.3 Comportamiento óptico de los materiales: hace relación a la interacción de los materiales con radiaciones electromagnéticas viajando en forma de ondas de partículas electromagnéticas denominadas fotones. 
Existen varias fuentes que emiten fotones, los cuales al interactuar con los materiales pueden generar efectos ópticos como la absorción, transmisión, reflexión, refracción y un comportamiento eléctrico (como el que se mencionó en los semiconductores). Algunas propiedades ópticas son generadas en el vidrio al colorearlo, mediante la infiltración de impurezas en su estructura, constituidas por óxidos metálicos. De esta manera, el vidrio adquiere un color que puede ser ámbar o verde y que protege a las bebidas que pueda contener el recipiente (como en la cerveza, medicamentos y algunas gaseosas) de los efectos de la luz solar.
2.4 Comportamiento térmico de los materiales: los materiales al ser expuestos a cambios de temperatura, sufren alteraciones apreciables en sus propiedades mecánicas, físicas y químicas. Algunos de ellos conducen muy lentamente el calor a pesar de estar expuestos a elevadas temperaturas, proporcionándoles excelentes aplicaciones como aislantes tér. micos; por lo cual son conocidos como ma. teriales refractarios. Además requieren grandes cambios de temperatura para modificar de manera apreciable sus propieda. des, lo que les confiere una buena estabilidad térmica.

\section{COMPORTAMIENTO DE LOS MATERIALES}

En la siguiente tabla se resume el comportamiento y algunas de las aplicaciones corrientes de los materiales metálicos, cerámicos y poliméricos.

\begin{tabular}{|l|l|l|}
\hline TIPO DE MATERIAL & PROPIEDADES & APLICACIONES \\
\hline & Resistencia a lá tracción: elevada & $\begin{array}{l}\text { Utilizados para la construcción de maquinaria, } \\
\text { estructuras, obras civiles. }\end{array}$ \\
\cline { 2 - 3 } & Rigidez: grande & Construcción de vigas estructurales. \\
\cline { 2 - 3 } METALES Y SUS & $\begin{array}{l}\text { Ductilidad: se puede controlar } \\
\text { mediante procesos de fabricación }\end{array}$ & $\begin{array}{l}\text { Fabricación de ejes para la transmisión de } \\
\text { potencia, refuerzo estructural en obras civiles }\end{array}$ \\
\cline { 2 - 3 } & $\begin{array}{l}\text { Dureza: tienen una gran dureza } \\
\text { comparada con los otros materiales }\end{array}$ & $\begin{array}{l}\text { Utilizados en una gran cantidad de } \\
\text { Herramientas de corte, y elementos sometidos } \\
\text { a fricción. }\end{array}$ \\
\cline { 2 - 4 } & $\begin{array}{l}\text { Comportamiento eléctrico: buenos } \\
\text { conductores eléctricos }\end{array}$ & $\begin{array}{l}\text { Fabricación de cables para la conducción de } \\
\text { energía eléctrica }\end{array}$ \\
\cline { 2 - 3 } & $\begin{array}{l}\text { Comportamiento magnético: generan y y } \\
\text { modifican campos magnéticos }\end{array}$ & $\begin{array}{l}\text { Ideales para la implementación de imanes } \\
\text { temporales o permanentes. }\end{array}$ \\
\cline { 2 - 3 } & $\begin{array}{l}\text { Comportamiento óptico: son opacos } \\
\text { que evita que transmitan la luz. }\end{array}$ & $\begin{array}{l}\text { Tienen pocas aplicaciones desde el punto de } \\
\text { vista de la óptica }\end{array}$ \\
\cline { 2 - 3 } & $\begin{array}{l}\text { Comportamiento térmico: buenos } \\
\text { conductores de calor. }\end{array}$ & $\begin{array}{l}\text { Utilizados para la elaboración de resistencias } \\
\text { eléctricas para las estufas, intercambiadores de } \\
\text { calor. }\end{array}$ \\
\hline
\end{tabular}




\begin{tabular}{|c|c|c|}
\hline \multirow{7}{*}{ CERÁMICOS } & Resistencia a la tracción: elevada & Base constructiva de edificaciones. \\
\hline & Rigidez: grande. & $\begin{array}{l}\text { Para construir vigas sometidas a grandes } \\
\text { cargas. }\end{array}$ \\
\hline & Dureza: elevada & Fabricación de herramientas de corte. \\
\hline & $\begin{array}{l}\text { Comportamiento eléctrico: a } \\
\text { temperatura ambiente se } \\
\text { comportan como aislantes } \\
\text { eléctricos pero a temperaturas } \\
\text { inferiores a los }-170^{\circ} \mathrm{C} \text {. Se } \\
\text { comportan como } \\
\text { superconductores. }\end{array}$ & $\begin{array}{l}\text { En equipos destinados a estudios de } \\
\text { medicina que trabajan con campos } \\
\text { electromagnéticos se aprovecha su } \\
\text { comportamiento como superconductor. }\end{array}$ \\
\hline & $\begin{array}{l}\text { Comportamiento magnético: no } \\
\text { generan ni afectan los campos } \\
\text { magnéticos a temperatura } \\
\text { ambiente. }\end{array}$ & $\begin{array}{l}\text { Cuando adquieren el comportamiento } \\
\text { como superconductores pueden generar } \\
\text { grandes campos electromagnéticos. }\end{array}$ \\
\hline & $\begin{array}{l}\text { Comportamiento óptico: algunos } \\
\text { cerámicos (vidrios) son } \\
\text { transparentes con lo cual permiten } \\
\text { el paso (y desviación) de la luz. }\end{array}$ & $\begin{array}{l}\text { Construcción de microscopios, lentes y } \\
\text { aparatos ópticos en general. }\end{array}$ \\
\hline & $\begin{array}{l}\text { Comportamiento térmico: son } \\
\text { aislantes térmicos por excelencia. }\end{array}$ & $\begin{array}{l}\text { Recubrimientos de hornos, fabricación de } \\
\text { partes que funcionan a temperaturas } \\
\text { sumamente elevadas. }\end{array}$ \\
\hline \multirow{7}{*}{ POLIMEROS } & $\begin{array}{l}\text { Resistencia a la tracción: baja } \\
\text { comparada con los metales y } \\
\text { cerámicos }\end{array}$ & \\
\hline & $\begin{array}{l}\text { Rigidez: en la gran mayoría de los } \\
\text { polímeros es muy baja, pero } \\
\text { existen algunos tipos como los } \\
\text { termoendurecibles que logran una } \\
\text { buena rigidez. }\end{array}$ & $\begin{array}{l}\text { Con bastante frecuencia son reforzados } \\
\text { con fibras (transformándose en materiales } \\
\text { compuestos) para mejorar su rigidez, } \\
\text { encontrando así aplicación en la } \\
\text { fabricación de partes para automóviles, } \\
\text { tanques para almacenar agua, tinas, etc }\end{array}$ \\
\hline & Ductilidad: son bastante ductiles: & $\begin{array}{l}\text { Apropiados para fabricar correas para la } \\
\text { transmisión de movimiento, }\end{array}$ \\
\hline & $\begin{array}{l}\text { Dureza: comparada con los otros } \\
\text { materiales es bastante baja. }\end{array}$ & \\
\hline & $\begin{array}{l}\text { Comportamiento eléctrico: son } \\
\text { aislantes eléctricos. }\end{array}$ & $\begin{array}{l}\text { Son utilizados para el recubrimiento de } \\
\text { cables destinados a la conducción } \\
\text { eléctrica, como base para la fabricación de } \\
\text { circuitos integrados y para todo tipo de } \\
\text { situación donde aislar la electricidad se } \\
\text { hace necesario. }\end{array}$ \\
\hline & $\begin{array}{l}\text { Comportamiento óptico: los hay } \\
\text { opacos y transparentes. }\end{array}$ & $\begin{array}{l}\text { Los que son transparentes son utilizados } \\
\text { para la fabricación de instrumentos } \\
\text { ópticos. También reemplazan en algunas } \\
\text { aplicaciones arquitectónicas los vidrios } \\
\text { tradicionales. }\end{array}$ \\
\hline & $\begin{array}{l}\text { Comportamiento térmico: son muy } \\
\text { susceptibles al calor. }\end{array}$ & $\begin{array}{l}\text { Permiten la fabricación de piezas con } \\
\text { formas bastante complejas mediante la } \\
\text { aplicación de calor y presión lo que se } \\
\text { aprovecha para fabricar tableros para } \\
\text { automóviles por ejemplo }\end{array}$ \\
\hline
\end{tabular}




\section{CONCLUSIONES}

- Para cualquier campo de la ingeniería (especialmente en el diseño y la fabricación) es importante tener un amplio conocimiento de la gran variedad de materiales y de sus propiedades, ya que esto permite seleccionar el material más apropiado para alguna aplicación particular, teniendo en cuenta aspectos como la disponibilidad del mismo, aspectos económicos, ambientales, entre otros.

- El estudio de los materiales es una ciencia de gran importancia y complejidad, que es necesario abordar para lograr mejores avances en el desarrollo tecnológico y alcanzar así mejores niveles de vida.
- Se hace igualmente importante, mediante proyectos de investigación, la generación de nuevos materiales, con mejores característi. cas que los ya existentes, y que proporcio. nen ventajas en su utilización.

\section{BIBLIOGRAFÍA}

1. SMITH, William F. "Fundamentos de la Ciencia e Ingeniería de Materiales". Editorial Mc. Graw Hill, tercera edición. Madrid, 1996.

2. BRADY, George,CLAUSER, Henry, \& Vaccari John. "Materials Handbook". Fourteenth Edition. Editorial Mc Graw Hill, 1996.

3. DOWLING Norman, "Mechanical Behavior of Materials: Engineering Methods for Deformation, Fracture, and Fatigue" Editorial Prencite Hall. Segunda Edición, 1998.

4. http://www.chms.ucdavis.edu

5. http://www.cmse.ed.ac.uk/

6. http://www.msm.cam.ac.uk/ 